\title{
Evaluación de la calidad de un medio urbano: la ciudad de Teruel
}

\author{
M. ${ }^{a}$ Pilar Borderías Uribeondo y Manuel Garcia Márquez
}

\begin{abstract}
RESUMEN
ABSTRACT

El desarrollismo y la ausencia de una política urbana orientada a mejorar las condiciones de vida en las ciudades, han ocasionado numerosos problemas urbanísticos y un importante deterioro de la calidad del medio urbano. En general, los estudios de la calidad ambiental se han centrado en la gran ciudad, siendo menor la atención prestada a la valoración ambiental de las pequeñas ciudades, donde los problemas urbanos también son motivo de gran preocupación. Este artículo, a partir de las características de los distintos elementos que configuran el medio ambiente de Teruel y tomando como unidad de análisis los barrios, homogéneos en si mismos y heterogéneos entre si, llega a una valoración urbanística y ambiental de esta pequeña ciudad, mediante la aplicación de diversos criterios de valoración ambiental utilizados en otros estudios similares.

Unimpeded development and the absence of an urban policy oriented to improve living conditions in the cities, have caused many urbanistic problems and an important impairment of the urban medium. In general, studies on enviromental quality have been focused on the big city, paying a lesser attention to environmental evaluation in small town, Teruel, through the application of different environmental evaluation criteria used in similar studies. The evaluation is considered from the characteristics of the different elements which shape Teruel environment and takes the districts, homogeneous in themselves and heterogeneous among themselves as a unit of analysis.
\end{abstract}




\section{INTRODUCCIÓN}

Es opinión común que la mejor política del medio ambiente es la que evita los problemas medioambientales antes de que se produzcan, teniendo en cuenta las repercusiones que tendrá la implantación de una determinada actividad sobre el medio. En muchos casos ya no estamos en condiciones de hacerlo y sólo nos queda valorar los daños ocasionados e intentar mejorar los espacios que se han deteriorado.

La ciudades, que son consideradas como una manifestación del desarrollo y del bienestar, configuran un medio que plantea diversos inconvenientes ambientales. Si bien son las grandes ciudades las que más preocupación suscitan, al variar la incidencia de los problemas urbanos en relación con el tamaño y estructura de la ciudad, sus actividades o funciones y su grado de desarrollo, no por ello, las pequeñas ciudades quedan exentas de éstos.

El desarrollismo y la ausencia de una política urbana orientada a mejorar las "condiciones de vida" hasta la década de los 80 , fueron los causantes de las carencias de la calidad urbana en nuestros días, tanto en las grandes como en las pequeñas ciudades. A partir de ese momento, y debido, en gran parte, al empuje de los movimientos asociativos vecinales (que reivindicaban más y mejores dotaciones colectivas, la creación o mejora de infraestructuras deficitarias como urbanización y alumbrado público, control de actividades contaminantes, y la protección y defensa del patrimonio y de los elementos naturales de calidad), la política sobre la ciudad española empezó a realizar medidas sectoriales, plasmadas en intervenciones concretas en las áreas residenciales.

La ciudad de Teruel, a pesar de ser una pequeña y tranquila capital de provincia, con una función basicamente administrativa, presenta determinados problemas urbanos que reducen su calidad medioambiental y que hemos querido valorar. Ya en 1976, Fernando Chueca Goitia hizo una valoración del grado de deterioro urbano de las capitales de provincia españolas en su libro "La destrucción del legado urbanístico español". En esta valoración, con índices entre 1 y 10, la ciudad de Teruel quedaba en un término medio, con un índice 6 , en el puesto 20 de las ciudades más deterioradas. No obstante, la imagen que produce Teruel a este autor en este año, está lejos de ser la de una ciudad de deterioro medio puesto que, en su descripción, emplea términos tan duros como que la ciudad produce un efecto triste y melancólico, o que en la ciudad vieja se ve por todas partes decrepitud y desidia. Así dice de ella: 
«El Estado nacional tuvo a gala llevar a cabo una restauración a fondo y sin escatimar medios en honor de la ciudad primero perdida y luego reconquistada... pero pasada la fiebre de aquellos años reconstructores en los que intervino Regiones Devastadas... la ciudad de Teruel volvió a quedar abandonada y hoy produce un efecto más bien triste y melancólico". "Como la topografía de Teruel aisla la ciudad vieja al estar colocada en una altura sobre los niveles bajos por donde corre el río Turia, su estructura urbana y su caserío se ha conservado en líneas generales, aunque por todas partes veamos decrepitud y desidia. Las edificaciones modernas de los arrabales son, en general, lastimosas y de una calidad ínfima, el estado de la urbanización deplorable". Y finalizaba su valoración diciendo: "esperamos mejores tiempos que saquen a Teruel de su actual postración".

Pasados veinte años de estas afirmaciones, no parece que la ciudad antigua haya mejorado mucho su imagen.

\section{LOS ELEMENTOS DEL MEDIO AMBIENTE}

Al observar la ciudad percibimos un conjunto de elementos tanto naturales como humanos, que, estrechamente interrelacionados, configuran el medio ambiente urbano de Teruel. Las caracteristicas de los medios que estos elementos configuran y cuanto sucede en ellos influye en la calidad de la vida de todas las personas que se reunen en la comunidad urbana. Los elementos naturales, relieve, aire, agua y vegetación, son la base para la vida y el asentamiento urbano, al tiempo que son los principales afectados por la acción antrópica. Por su parte, los elementos humanos como morfología, usos del suelo, equipamientos y relación social son los que confieren a la ciudad sus características específicas. Todos ellos serán considerados en la evaluación de la calidad medioambiental de Teruel.

- El relieve es un elemento importante del paisaje urbano y también del desarrollo físico de Teruel. Los ríos Alfambra y Guadalaviar o Turia, y los barrancos y ramblas, constituyen hitos naturales importantes; éstos, con su acción erosiva, han dejado en resalte los altiplanos que dan albergue tanto al Casco Antiguo como al desarrollo de una parte de la nueva ciudad. A ello hay que añadir las características de sus abruptas vertientes y sus valles que le confieren una imagen individualizadora. La calidad paisajística de Teruel tendria en su relieve un buen aliado de no ser por el descuido que afecta a sus vertientes, que presentan en gran parte un relieve ruinoso de cárcavas y abarrancamientos de arcillas, 10 que impide que sea un factor positivo a tener en cuenta al valorar la calidad ambiental. 
En la actualidad puede asignarsele al relieve una función simbólica, un significado de preeminencia o superioridad, que se traduce en un estatus social, seguridad, intimidad, además de un contacto con la naturaleza en el caso de relieve no totalmente urbanizado. Según Cullen (1974), este argumento simbólico funciona en la localización de determinadas y selectivas urbanizaciones residenciales, y así ocurre en Teruel, donde barrios situados en posiciones altas y aisladas, como el Pinar de la Muela y las Viñas, albergan las edificaciones de mejor calidad y pertenecientes a familias de elevado estatus económico-social, mientras que los situados en zonas bajas, como Arrabal, San Julián y los Arcos, o en escarpes, como las Cuevas del Siete, se configuran con viviendas de baja calidad y acogen a las clases de menor nivel socioeconómico.

La topografía ha sido uno de los indicadores tomados en la posterior valoración ambiental, considerándose más favorables las zonas altas por su simbolismo, posibilidad de perspectivas de paisaje y por su mayor facilidad de ventilación frente a la contaminación.

- Tanto las condiciones atmosféricas como la calidad del aire que se relaciona con ellas y con las actividades urbanas, son muy importantes en la valoración del medioambiente urbano. Frente a sus características climáticas generales, nos interesa destacar las peculiaridades microclímaticas que crean los distintos componentes urbanos. Aunque de poca intensidad, está confirmada la existencia de la isla de calor en el centro de Teruel (Cuadrat, 1992), en torno a $2{ }^{\circ} \mathrm{C}$ de diferencia con el extrarradio por término medio, que puede llegar a ser de $3^{\circ} \mathrm{C} 04^{\circ} \mathrm{C}$ o algo superior en días de inversión térmica. El Casco Histórico y el Ensanche configuran dos centros distintos de la isla de calor (primario y secundario respectivamente), mientras que las zonas más bajas de la ciudad, en momentos de situación anticiclónica, se convierten en zonas de embolsamiento de aire frío.

Además de modificar el clima, la actividad urbana también contamina el aire, aunque el problema real de estas emisiones se presenta cuando su volumen supera la capacidad de asimilación del medio sobre el que se vierten. En el caso de Teruel no creemos que ésta registre valores elevados. A pesar de su importancia, esta variable no ha sido valorada por no contar con valores cuantitativos para las unidades de análisis, aunque se relaciona de modo muy directo con las modificaciones climáticas que sí se han valorado. En cuanto a estas últimas, se ha tenido en cuenta la extensión y el grado en que afecta la isla de calor, por estar relacionada con la mayor densidad y actividad urbana, y consecuentemente, como hemos dicho, también con la mayor contaminación. Así, hemos considerado de más valor aquellas zonas en que no se veía modificada la temperatura. 
- Tanto la cantidad como la calidad del agua representan una fuente de vida y de desarrollo económico, al tiempo que es el vehículo más utilizado para la evacuación de deshechos, tanto de la comunidad humana como de sus distintas actividades, lo que da lugar a su degradación. Los ríos de Teruel, que tienen una impronta muy significativa en su paisaje, recogen las aguas residuales de la ciudad, que son depuradas a lo largo del río Turia hasta el embalse del Generalísimo.

De los recientes estudios realizados sobre la calidad de las aguas de los ríos Guadalaviar, Algambra y Turia, se puede deducir que, hasta su llegada a la ciudad, en general son de buena calidad. No obstante, para el abastecimiento urbano de la ciudad, dadas la mayor demanda y exigencias de salubridad, se instaló, a finales de la década de los ochenta, una planta potabilizadora, que proporciona una calidad homogénea y aceptable a las distintas unidades de análisis.

A su paso por Teruel, las aguas sufren un deterioro considerable, como consecuencia de los vertidos industriales del Polígono La Paz y urbanos de la ciudad. Deterioro que se manifiesta en un aumento de la temperatura y conductividad; en un incremento de los cloruros, materia orgánica y coliformes fecales; y en concentraciones muy elevadas de metales muy tóxicos - plomo y mercurio principalmente-. De hecho, el río Turia, aguas abajo de la ciudad, produce una notable pestilencia. El impacto ambiental es tal, que ha originado manifestaciones periódicas y la creación de una asociación de defensa del río.

- La vegetación es de gran valor para la calidad ambiental, tanto que la incorporación de las zonas verdes es una de las aportaciones mas significativas del urbanismo moderno para corregir los problemas derivados de procesos urbanizadores. A pesar de ello, éste es uno de los elementos que se ven mas gravemente atacados con la ubicación de la ciudad, hasta el punto que en ella sólo puede hablarse de replantación. Teruel cuenta con pocos espacios verdes públicos, de hecho más de la mitad de las unidades de análisis en la valoración ambiental no cuentan con la presencia de vegetación de caracter público, es decir, que formarían parte de la «miseria psico-física urbana" que denomina Philippe Saint Marc. Como zonas verdes urbanas pueden señalarse: el parque de los Fueros de Teruel, los jardines de la Ciudad Escolar, el jardín de la Glorieta, los jardincillos de la Escalinata frente a la estación del ferrocarril, un pequeño parque en el área de la Fuenfresca y arbolado en algunas calles del Ensanche, la carretera de Alcañiz, la carretera de Zaragoza y la Fuenfresca. En contraposición, las áreas de mayor calidad residencial, las Viñas y el Pinar de la Muela cuentan con abundante vegetación privada. 
Su presencia o ausencia ha sido uno de los indicadores que hemos tenido en cuenta en la valoración ambiental. Esta presencia o ausencia será matizada por la valoración de los servicios públicos de mantenimiento de parques y jardines, como veremos más adelante.

- La edificación construye un nuevo relieve tanto por la elevación de los edificios como por su disposición y distribución espacial; de modo que la forma o aspecto físico de la ciudad se hace patente, principalmente, a partir de dos componentes: el trazado viario y la edificación.

El trazado viario confiere a Teruel una morfología radioconcéntrica, adaptada a la topografía irregular del entorno y tomando unos ejes radiales a lo largo de las principales redes de comunicación (carreteras de Zaragoza, Sagunto y Alcañiz). Los barrios de época preindustrial como el Casco Histórico y sus ampliaciones del Arrabal, San Julián y Los Arcos, tienen calles estrechas y tortuosas. Por el contrario, la zona del Ensanche sigue la característica configuración geométrica de estas zonas de expansión urbana, adaptada a las necesidades de desplazamiento del momento, lo mismo que las nuevas urbanizaciones del área de la Fuenfresca, que ha supuesto la zona de mayor desarrollo urbanistico de los últimos años.

La edificación presenta distintas tipologías y alturas. La altura de las edificaciones, reflejo de la especulación del suelo en las ciudades, no llega a ser alarmante en Teruel, pues no está demasiado generalizado el desarrollo de altos edificios (la torre de Teruel en la Avenida Ruiz Jarabo, con 13 plantas, y algunas otras edificaciones de más de 10 plantas en el Ensanche), no obstante, en un sentido relativo, los edificios que alcanzan esta altura presentan un vivo contraste paisajístico con las edificaciones más bajas con las que se entremezclan.

La tipología edificatoria de Teruel es heterogénea y presenta contrastes y falta de coherencia en las unidades de estudio. Viviendas unifamiliares aisladas y adosadas, plurifamiliar de manzanas cerradas y plurifamiliar de bloques aislados son las tipologías que hemos valorado.

Las viviendas unifamiliares aisladas presentan una gran diversidad, desde viviendas unifamiliares aisladas de calidad en las Viñas y el Pinar de la Muela (en algunos casos constituyen viviendas de segunda residencia), hasta viviendas de una o dos plantas de tipo rural y baja calidad en los barrios del Arrabal, San Julián, los Arcos, el Carmen, carretera de Alcañiz y carretera de Zaragoza. Un caso extremo lo constituyen las cuevas del barrio las Cuevas del Siete. En el Ensanche también se combinan viviendas unifamiliares de tipo chalé con otras tipologías. 
Las viviendas unifamiliares adosadas coinciden con el crecimiento periférico de las últimas décadas, que está configurando por todo el país amplias áreas residenciales para la clase media, este tipo se encuentra en las nuevas edificaciones de la Fuenfresca.

La vivienda plurifamiliar de manzanas cerradas es muy diversa. El "Grupo Generalisimo» constituye una urbanización de casas baratas en la carretera de Alcañiz, al igual que las del barrio de la Grama, y la urbanización de Cáritas en el barrio de San Julián. En el Carmen encontramos manzanas de 5 y 6 plantas en la parte más nueva del barrio y con algo mejor calidad que las deficientes casas bajas del conjunto, y de tipo rural de 2 ó 3 plantas en el Arrabal y San Julián. En el Ensanche las manzanas quedan constituidas por casas de 4 y 5 plantas. En el Casco Histórico se mantiene la fisonomía de ciudad antigua, en la que se entremezclan ruinosas edificaciones con urbanizaciones más recientes que rompen el conjunto urbanístico.

La vivienda plurifamiliar de bloques aislados se encuentra sobre todo en el segundo Ensanche. Estos bloques presentan diversas características y distintas alturas, desde grupos de 4, 8, ó 10 plantas, hasta de 13, como la Torre de Teruel. En la carretera de Alcañiz también se elevan edificios aislados de hasta 9 plantas.

La construcción de viviendas responde a las necesidades sociales y culturales a lo largo de la historia de la ciudad, y está interrelacionada con otros elementos como la densidad de población, la renta per cápita, etc.; al mismo tiempo, el grado de urbanización es un importante indicador de presión indirecto sobre el medio ambiente. Por ello hemos considerado que tanto la morfología como la tipología son indicadores a considerar en nuestra valoración.

- La función básicamente administrativa de Teruel hace que los problemas ambientales derivados de sus actividades no sean graves, aunque, dentro de la misma ciudad, hay que diferenciar entre las zonas de función residencial, las comerciales y las industriales. En los distintos planes urbanísticos de Teruel, se aprecia la influencia de la teoría de la ciudad funcional apoyada en el principio del "zoning", que divide la ciudad en zonas a las que asigna un uso, densidad y tipología edificatoria. La aplicación de estos principios da lugar, entre otras consecuencias, a la concentración de actividades, a la separación de grupos sociales, al aumento del tráfico urbano, etc.

- En el espacio residencial, la confluencia de contenidos físicos, sociales y económicos crea un microambiente en el que se refleja la calidad ambiental y tiene una gran influencia sobre la salud y la calidad de vida. 
Desde el punto de vista físico, el espacio residencial se estructura en un mosaico de áreas suficientemente homogéneas en sí mismas y heterogéneas entre sí. En Teruel el centro es muy heterogéneo; frente a barrios antiguos que contienen viviendas deterioradas y que albergan a una población marginal en muchos casos, se encuentran áreas que han sido renovadas, donde se encuentran clases sociales de mayor nivel de renta. A lo largo de las carreteras radiales se han ido creando asentamientos residenciales hasta llegar a las áreas de desarrollo periférico, que configuran igualmente espacios de gran heterogeneidad.

Desde el punto de vista social, la segregación espacial o separación residencial de subgrupos es un hecho, tanto en el caso de las clases de menor renta como en el caso de las de rentas superiores. El primer movimiento interno de población se produce con el primer Ensanche, asentándose en él parte de las clases adineradas, formandose así un primer espacio diferenciado respecto al conjunto del Casco Antiguo. Los propios documentos de planeamiento señalan la imagen de fragmentación de las áreas residenciales que se proponían en el Proyecto Parcial de Reforma Interior de Regiones Devastadas. En él se trazaban unas líneas de crecimiento basadas en criterios sociales como "barriada de agricultores", "bloques para obreros industriales", «zona residencial para funcionarios»,etc., como ocurre con las casas ultrabaratas en la carretera de Alcañiz - hoy desaparecidas- o las posteriores casas baratas del Grupo Generalísimo y barrio de la Grama; el caracter social de estas construcciones contrasta con la paralela formación de un nuevo barrio residencial, el Pinar de la Muela, al otro lado de la Vega, formado por viviendas unifamiliares tipo chalé de utilización veraniega. Igualmente, en los años 80 se produjo el auge de un mercado residencial espacialmente diferenciado con casas adosadas, pareadas 0 aisladas, y de vivienda unifamiliar en el área de la Fuenfresca.

Desde el punto de vista funcional sucede lo mismo que en el conjunto de las ciudades históricas españolas, en las que, a partir de 1960, el concepto de barrio se difumina y las tres actividades que definen un barrio, vivienda, trabajo y diversión, se separan y reparten por distintas zonas de la ciudad, la construcción de la Ciudad Escolar es un buen reflejo de ello. Así, mientras que las actividades de consumo, religiosas, de negocios y culturales hay que realizarlas en la ciudad histórica, que ha adquirido un caracter de centralidad, los nuevos espacios periféricos, como la Fuenfresca, han cambiado el significado de la ciudad, son dependientes y sin autonomía vital.

En la valoración final hemos considerado los niveles socioprofesionales de los habitantes de las unidades de análisis, puesto que consideramos 
que sus distintas exigencias y posibilidades económicas son una pauta que repercute notablemente en las condiciones de sus espacios de residencia. Al mismo tiempo, el propio mercado de la vivienda consigue establecer diferenciaciones a priori.

- El sector industrial en Teruel no es ni estable, ni boyante, ni dinámico sino que se caracteriza por la escasa diversidad industrial, por el predominio casi exclusivo de las actividades industriales de mantenimiento, de la construcción y sus anexas, por ser muy dependiente de la evolución del sector servicios, y por el reducido tamaño de las empresas.

Dadas estas características, no es de extrañar que se encuentre distribuido por diversas zonas de la ciudad, aunque con una tendencia a localizarse en áreas específicas de uso industrial como son el polígono industrial La Paz, la zona industrial de las Cerámicas y la zona de la Vega. Por el resto de la ciudad se alternan pequeñas industrias, en su mayor parte familiares. No se trata de una industria especialmente contaminante en cuanto a vertidos se refiere, al tiempo que tampoco está muy diversificada, por lo que en su valoración no se ha tenido en cuenta su actividad sino sólo su ubicación y concentración.

- Los servicios comerciales, educacionales, culturales, administrativos, bancarios, religiosos y sanitarios constituyen actividades que se concentran en un alto grado en zonas concretas, dándoles un valor específico. Son cuatro las zonas de concentración preferente de los servicios: el Casco Histórico, el Ensanche y las carreteras de Zaragoza y Alcañiz.

El Casco Histórico es el que concentra la mayor parte de los servicios de la ciudad y en el que se encuentran con mayor intensidad muchos de ellos. En él se localiza un comercio especializado, la actividad bancaria y la administración pública, en cambio, el comercio secundario se dispersa en el resto del espacio residencial, al tiempo que aparece una gran superficie comercial en el extrarradio.

El espacio educacional se concentra en la Ciudad Escolar -carretera de Alcañiz-, en la que se encuentran representados todos los niveles de educación. No obstante, no faltan algunos centros dispersos por distintos barrios, sobre todo en el Ensanche donde en su mayoría son de enseñanza privada.

En el Ensanche los servicios se disipan más entre el uso residencial pero destacan los correspondientes a la Administración pública, educación, asistencia sanitaria y asistencia social. Espacios de usos significativos aparecen en la carretera de Zaragoza - Hospital Provincial, Asilo y Prisión Provincial—; en la Vega - Estación de Ferrocarril-; en el Pinar de 
la Muela -Sanatorio Psiquiátrico-; en los Arcos -Cementerio-; y en la Fuenfresca - hipermercado-.

- Como equipamientos urbanos hemos considerado el conjunto de servicios ofrecidos a los ciudadanos de forma directa por el Municipio. Los equipamientos de la ciudad constituyen un importante indicador para valorar la calidad ambiental: la pavimentación, la iluminación, el abastecimiento de agua, el alcantarillado, la depuración de aguas, y los vertederos de sólidos urbanos, tienen una marcada influencia en la calidad de vida, al tiempo que son un reflejo de ella. Otro equipamiento fundamental para la valoración ambiental son los espacios de ocio y zonas verdes, cuya distribución se ha mencionado al tratar la vegetación. Al haberse valorado su presencia o ausencia como componente del espacio natural, la valoración en el medio urbano se hace mediante la aplicación del servicio público en relación con el cuidado de las zonas verdes.

\section{VALORACIÓN DE LA CALIDAD URBANISTICA Y AMBIENTAL}

El fenómeno urbano puede simplificarse como una oferta de bienestar, que depende de la calidad de su medio ambiente. A la hora de valorar la calidad del medio y la calidad de vida de los ciudadanos hay que tener en cuenta que se trata de un concepto cambiante en el tiempo y en el contexto sociocultural. En cuanto a calidad se refiere, las características del medio ambiente urbano que se consideran necesarias tienden a ser altamente subjetivas, puesto que al afectar de forma diferente a las distintas personas, se produce una variación considerable en sus opiniones. Así, la calidad del medio está, en gran parte, en función de un proceso humano de filtración, la percepción, y, a su vez, ésta viene configurada por la experiencia del individuo de acuerdo con toda una gama de sistemas de comportamiento que entran en interacción con el medio ambiente.

Teniendo esto en cuenta, hemos tratado de objetivizar lo subjetivo, utilizando los elementos de los medios natural y humano, seleccionando aquellos indicadores que son más habituales en el nivel que pretendemos: una visión general de la ciudad.

Como unidades de estudio para esta valoración se han tomado los barrios de la ciudad ya que éstos presentan unas caracteristicas que les individualiza y diferencia entre sí desde un punto de vista histórico, morfológico-estructural, espacial, socioeconómico o funcional. 
La evaluación de la calidad ambiental de Teruel tiene por objeto conocer el grado de calidad relativa de cada una de las unidades analizadas y diferenciar espacialmente la calidad del medio urbano.

La valoración que obtengamos debe tener una interpretación cautelosa, puesto que si bien se han seleccionado aquellos indicadores que se basan en valores generales conocidos y que son buscados por lo general, como las manifestaciones y preferencias son libres, en ciertos casos pueden no coincidir con aquellas que normalmente determinan la conducta humana, como expondremos más adelante.

La asignación de los indicadores se relaciona con el concepto a valorar, y puesto que la relación entre el indicador y el concepto fundamental se define en términos de probabilidad y no de certeza, es necesario utilizar el mayor número posible de indicadores, a pesar de lo cual, la relación entre los indicadores seleccionados y el rasgo fundamental que tratan de medir jamás podrá ser una relación exacta. Hemos utilizado sobre todo indicadores del medio humano, puesto que el medio natural en la ciudad queda notablemente modificado, no obstante sí se han considerado algunos aspectos de él, como ya se ha indicado anteriormente.

Al contar sólo con variables cualitativas, se les ha asignado un valor cuantitativo en relación con apreciaciones más o menos subjetivas, que se describen más adelante. $A$ todas las variables se les ha asignado un valor de 1 a 3 y una ponderación según su importancia relativa.

Las condiciones del medio natural valoradas han sido:

- La topografía, considerándose más favorables las zonas altas por su simbolismo, posibilidad de perspectivas de paisaje y por su mayor facilidad de ventilación frente a la contaminación. Así se ha asignado un valor 1 a las zonas bajas, 2 a las medias y un 3 a las zonas altas.

- El microclima en relación con la isla de calor detectada, que indica indirectamente las distintas condiciones de ventilación, congestión y saneamiento ambiental, al tiempo que es el reflejo de las características morfológicas y de la intensidad de la actividad humana, que provocan la distorsión en el clima. Al haberse detectado como media una modificación de $2{ }^{\circ} \mathrm{C}$ entre el centro y la periferia, se ha asignado un valor 1 al núcleo central, un 2 al área con $1{ }^{\circ} \mathrm{C}$ menos, y un 3 al área que alcanza $2{ }^{\circ} \mathrm{C}$ menos que el núcleo central de la isla de calor.

- La vegetación, en relación con su grado de presencia o con su ausencia; en este último caso de ha dado un valor 0 . Se ha asignado un valor de 3 a los barrios que contaban con espacios públicos de vegetación diversificada (arbolado, parques, jardines), y a los espacios con abundan- 
te vegetación privada; un valor de 2 a los que contaban solamente con arbolado; y un valor 1 donde había una escasa vegetación.

Con respecto al medio humano hemos valorado:

- La tipología de la edificación, en la que se han diferenciado los siguientes tipos de inmuebles: unifamiliar aislado, unifamiliar adosado, plurifamiliar en manzanas cerradas y plurifamiliar en bloques aislados. Se calculó el porcentaje que representaban en cada unidad de análisis y se asigno la valoración según el tipo predominante. Al predominio de vivienda plurifamiliar, tanto en manzana cerrada como en bloque aislado se le asignó un valor 1; a la vivienda unifamiliar adosada un valor 2 y a la vivienda unifamiliar aislada un valor 3; de este grupo se ha segregado la vivienda unifamiliar de tipo rural, a la que se le ha dado valor 1 . En los casos en que se encontraban muy equilibradas viviendas unifamiliares y plurifamiliares se les asignó un valor de 1,5. En los casos de áreas mixtas de uso residencial e industrial, este último aspecto queda matizado al valorar negativamente la presencia del uso industrial.

- La altura de los edificios se ha agrupado en tres tipos, cada uno de ellos resultado de un intervalo de número de plantas: de baja altura (planta baja y baja más 1 planta); de altura media-baja (de planta baja más 2 plantas, a planta baja más 5 plantas); de altura media-alta (de planta baja más 6 a planta baja más 8 plantas). Aunque existen edificaciones con mayor número de plantas (hasta 13 plantas en la Torre de Teruel), no se ha establecido un intervalo superior, de altura alta (de planta baja más 9 plantas en adelante, según algunas metodologías de este indicador), por no constituir en ningún caso un porcentaje prioritario dentro de las unidades de análisis. A cada uno de ellos se le ha asignado un valor de 1 a 3 de mayor a menor altura.

- Los usos del suelo han sido valorados de la siguiente forma:

- Para el uso residencial se ha tenido en cuenta el nivel socioprofesional de sus habitantes: un 1 al nivel social de clases humildes, un 2 para las clases medias y un 3 para las clases altas. Y también, el estado exterior de los edificios, que se calificó bueno (valor 3) cuando había armonía y estética, es decir, terrazas bien cuidadas, toldos iguales, etc. y presentaban un buen mantenimiento; regular (valor 2) cuando las terrazas estuvieran sin cuidar, hubiera terrazas desiguales en cuanto a cerramiento, los toldos fueran distintos, etc.; y malo (valor 1) cuando las terrazas fuesen utilizadas como trasteros, hubiese ropa tendida, bombonas, la fachada estuviera deteriorada, etc.

- El uso industrial, que tiene unas connotaciones ambientales negativas, ha sido valorado con valores negativos en relación con su mayor 0 
menor presencia, pues el tipo de actividad no resultaba significativo en el grado de contaminación. Así, un valor -3 alcanzan las zonas de uso predominantemente industrial, un valor -2 las zonas en que se entremezcla con otras actividades, y un valor -1 donde apenas existe.

- Para equipamientos y servicios se han considerado distintas variables: iluminación, asfaltado, limpieza de las calles, cuidado de las zonas verdes, asignandose en cada caso un valor entre 1 y 3 según se hayan considerado las condiciones como mala, regular o buena respectivamente. Así, en relación con la iluminación, hemos considerado que era buena cuando existian suficientes farolas y todas tenían luz; era regular si sólo había algunas farolas y no todas tenian luz; y mala cuando no había farolas o éstas estaban sin luz. Para valorar los servicios se ha tenido en cuenta la actividad comercial asignándose un 3 cuando era abundante y diversificada, un 2 cuando no era abundante aunque fuera diversificada y no contara con comercios de alto rango, y un 1 cuando era escasa.

En cuanto al asfaltado, se consideró bueno si estaba en buen estado y sin baches; regular si el espacio estaba a medio asfaltar, con baches, o con tramos distintos o con cemento; y malo cuando las calles estaban sin asfaltar o con muchos baches. Asignándose, respectivamente, valores de 3 a 1.

El grado de limpieza de las calles se consideró bueno cuando estuviera todo limpio, sin papeles y sin olores; regular cuando hubiera algo de suciedad o papeles; y mala cuando se apreciara suciedad, basuras, malos olores, etc. Al igual que en el caso anterior se les dió valores de 3 a 1 de mayor a menor limpieza.

El cuidado de zonas verdes se calificó bueno, con un valor 3 , cuando existía una zona verde cuidada; regular, con un valor 2 , cuando la zona verde estaba poco cuidada, y mala, con un valor 1 , si las zonas verdes estaban descuidadas y pisoteadas. En este caso se añadió la valoración 0 para los casos en que no hubiera vegetación.

Antes de realizar la valoración global hemos creido interesante hacer sucesivas valoraciones parciales, pues, como deciamos al principio, si bien existe una correlación entre la calidad y los indicadores propuestos para la valoración, hay casos en nuestro análisis en que no se produce dicha correlación por circunstancias concretas, y distorsionan los resultados finales. Asi, se supone que un barrio de alta calidad en la viviendas y con habitantes de elevado nivel socioprofesional debería contar con unos buenos equipamientos, pués esto no sucede en el barrio del Pinar de la Muela por ejemplo. Por otro lado, ello nos permite, además de individualizar la calidad en distintos aspectos, sintetizar las variables finales, pues algu- 
nos de los indicadores tomados en las valoraciones parciales pueden interrelacionarse. De este modo, aseguramos que las variables de la valoración final tengan el mayor grado de independencia.

Siguiendo estos criterios, hemos valorado en primer lugar la calidad del medio urbano natural, dando un valor de ponderación de 0,45 a las zonas verdes, de 0,35 a las características microclimáticas, y de 0,20 a la topografía. La decisión de esta ponderación está relacionada con la gran preocupación que existe actualmente por la ubicación de zonas verdes, que son consideradas fundamentales para que la ciudad pueda dar respuesta a los problemas de contaminanción y de densificación, además, la vegetación es uno de los elementos más afectados por los asentamientos humanos. El microclima lo hemos dejado en segundo término porque si bien es una respuesta a la morfología, tipología y actividad urbanas, y uno de los elementos más comunmente valorados en los impactos medioambientales de la ciudad, se puede ver favorecido por la implantación de zonas verdes, con lo cual puede darse una relación con el valor anterior. En ultimo lugar hemos dejado la topografía por ser el elemento que ha cambiado menos, aunque influye en las características microclimáticas y en los valores simbólicos. Con ello se obtienen los valores del cuadro VI.1.

Cuadro VI.1. Valoración según indicadores del Medio Natural Urbanizado

\begin{tabular}{lllllcccc}
\hline $\begin{array}{l}\text { DISTRITOS/ } \\
\text { VARIABLES }\end{array}$ & 1 & 2 & 3 & $T$ & & & & \\
\hline C.Histórico & 3 & 1 & 1 & 5 & 0,60 & 0,35 & 0,45 & 1,40 \\
Arrabal & 1 & 2 & 0 & 3 & 0,20 & 0,70 & 0,00 & 0,90 \\
San Julián & 1 & 2 & 0 & 3 & 0,20 & 0,70 & 0,00 & 0,90 \\
$1^{\text {er Ensanche }}$ & 3 & 2 & 3 & 8 & 0,60 & 0,70 & 1,35 & 2,65 \\
$2^{\text {Q Ensanche }}$ & 3 & 2 & 3 & 8 & 0,60 & 0,70 & 1,35 & 2,65 \\
Los Arcos & 1 & 2 & 0 & 3 & 0,20 & 0,70 & 0,00 & 0,90 \\
Cta Alcañiz & 3 & 3 & 1 & 7 & 0,60 & 1,05 & 0,45 & 2,10 \\
Carmen/Cuev & 2 & 2 & 0 & 4 & 0,40 & 0,70 & 0,00 & 1,10 \\
CtrZaragoza & 2 & 3 & 2 & 7 & 0,40 & 1,05 & 0,90 & 2,35 \\
C. Escolar & 3 & 3 & 3 & 9 & 0,60 & 1,05 & 1,35 & 3,00 \\
Las Viñas & 3 & 3 & 3 & 9 & 0,60 & 1,05 & 1,35 & 3,00 \\
P. Muela & 3 & 3 & 3 & 9 & 0,60 & 1,05 & 1,35 & 3,00 \\
B Jorgito & 2 & 3 & 0 & 5 & 0,40 & 1,05 & 0,00 & 1,45 \\
La Vega & 1 & 3 & 2 & 6 & 0,20 & 1,05 & 0,90 & 2,15 \\
Fuenfresca & 3 & 3 & 3 & 9 & 0,60 & 1,05 & 1,35 & 3,00 \\
P.Industria & 2 & 3 & 0 & 5 & 0,40 & 1,05 & 0,00 & 1,45 \\
C. San Blás & 2 & 3 & 0 & 5 & 0,40 & 1,05 & 0,00 & 1,45 \\
Cerámicas & 1 & 3 & 0 & 4 & 0,20 & 1,05 & 0,00 & 1,25 \\
\hline
\end{tabular}

Variables: 1) topografía; 2) microclima; 3)presencia de vegetación. 
Los resultados obtenidos se han agrupado en tres intervalos:
De 0 a 1,5 baja calidad
De 1,6 a 2,5 . calidad media
De 2,6 a 3 alta calidad

Así tenemos que en orden de menor a mayor calidad del medio natural se encuentran: el Arrabal, San Julián, Los Arcos, el Carmen y las Cuevas del Siete, las Cerámicas, el Casco Histórico, el barrio del Jorgito, el Polígono Industrial y la carretera de San Blás, de calidad baja; la carretra de Alcañiz, la Vega, y la carretera de Zaragoza, con una calidad media; y el Ensanche, la Ciudad Escolar, las Viñas, la Muela y la Fuenfresca de alta calidad (Figura 1).

Para valorar la calidad de la edificación hemos utilizado la tipología de la edificación, su altura y el aspecto exterior de las viviendas. Los valores de ponderación que se han aplicado han sido de $0,40,0,25$ y 0,35 respectivamente. Hemos considerado la tipología en primer lugar por ser, de entre los tres, el elemento que más repercusión tiene en la configuración del paisaje urbano; la altura se ha dejado en tercer lugar por ser en cierto modo una matización del anterior; y en segúndo lugar hemos considerado el aspecto exterior, por ser el que da una imagen de la calidad de la vivienda. Aunque en el cuadro aparecen calculados sus valores, los barrios correspondientes al Polígono Industrial, la Carretera de San Blás, y las Cerámicas, no deben considerarse en su estricto valor por tratarse de zonas que no tienen un uso residencial, y, por tanto, los valores aportados hacen referencia a los edificios de usos industriales.

Con estos valores hemos obtenido los resultados del cuadro VI.2.

Cuadro VI.2. Valoración según indicadores de edificación

\begin{tabular}{lllllllll}
\hline $\begin{array}{l}\text { DISTRITOS/ } \\
\text { VARIABLES }\end{array}$ & 1 & 2 & 3 & $T$ & & & \\
\hline C. Histórico & 1 & 2 & 2 & 5 & 0,40 & 0,50 & 0,70 & 1,60 \\
Arrabal & 1 & 2 & 1 & 5 & 0,40 & 0,50 & 0,35 & 1,25 \\
San Julián & 1 & 2 & 2 & 5 & 0,40 & 0,50 & 0,70 & 1,60 \\
1 $^{\text {er Ensanche }}$ & 1,5 & 2,5 & 3 & 7 & 0,60 & 0,62 & 1,05 & 2,27 \\
$2^{o}$ Ensanche & 1 & 2 & 2 & 5 & 0,40 & 0,50 & 0,70 & 1,60 \\
Los Arcos & 1 & 2 & 2 & 5 & 0,40 & 0,50 & 0,70 & 1,60 \\
Ctra Alcañiz & 1 & 2 & 2 & 5 & 0,40 & 0,50 & 0,70 & 1,60 \\
Carmen/Cueva & 1 & 2 & 2 & 5 & 0,40 & 0,50 & 0,70 & 1,60 \\
Ctr Zaragoza & 1 & 2 & 2 & 5 & 0,40 & 0,50 & 0,70 & 1,60 \\
C. Escolar & 1 & 2 & 3 & 6 & 0,40 & 0,50 & 1,05 & 1,95 \\
\hline
\end{tabular}




\section{VALORACIÓN MEDIOAMBIENTAL DE DIVERSOS COMPONENTES URBANOS}
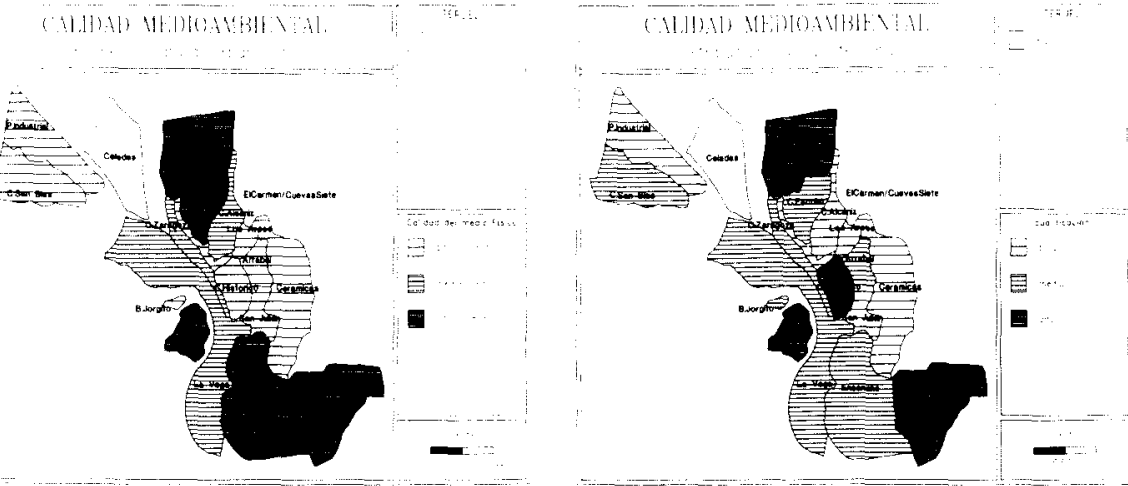

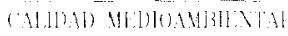

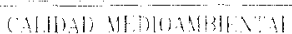

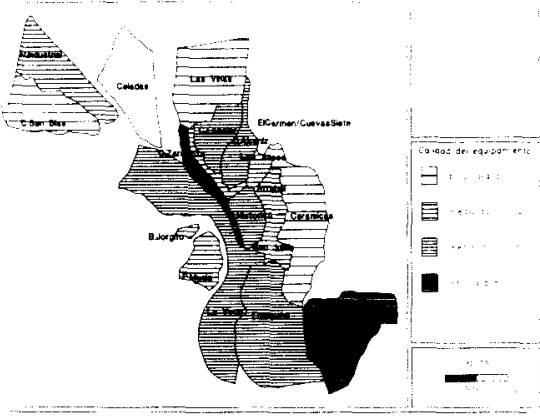

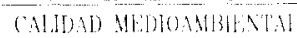

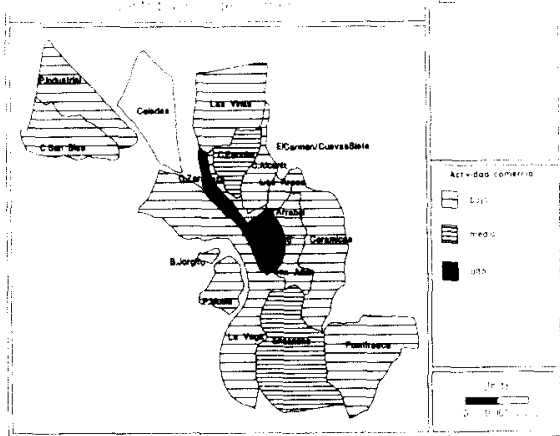

Figura 1. La valoración individualizada de distintos componentes del medio urbano muestra la heterogeneidad tanto en un barrio como entre todos los que configuran la ciudad. 
Cuadro VI.2. Valoración según indicadores de edificación (continuación)

\begin{tabular}{lllllcccc}
\hline $\begin{array}{l}\text { DISTRITOS/ } \\
\text { VARIABLES }\end{array}$ & 1 & 2 & 3 & $T$ & & & & \\
& & & & & 1 & 2 & 3 & TOTAL \\
\hline Las Viñas & 3 & 3 & 2 & 8 & 1,20 & 0,75 & 0,70 & 2,65 \\
P. Muela & 3 & 3 & 3 & 9 & 1,20 & 0,75 & 1,05 & 3,00 \\
B Jorgito & 1 & 2,5 & 2 & 5,5 & 0,40 & 0,62 & 0,70 & 1,72 \\
La Vega & 2 & 3 & 3 & 8 & 0,80 & 0,75 & 1,05 & 2,60 \\
Fuenfresca & 2 & 3 & 3 & 8 & 0,80 & 0,75 & 1,05 & 2,60 \\
P.Industrial & 1 & 3 & 2 & 6 & 0,40 & 0,75 & 0,70 & 1,85 \\
C San Blás & 3 & 2 & 3 & 8 & 1,20 & 0,50 & 1,05 & 2,75 \\
Cerámicas & 1 & 2 & 1 & 4 & 0,40 & 0,50 & 0,35 & 1,25 \\
\hline
\end{tabular}

Variables: 1) tipologia de la edificación, 2) altura de edificación, 3) aspecto exterior de las viviendas.

Los resultados obtenidos se han agrupado en cuatro intervalos:
De 0 a 1,5 baja calidad
De 1,6 a 2 calidad media-baja
De 2,1 a 2,5 calidad media-alta
De 2,6 a 3 alta calidad

Así, tenemos el Arrabal y las Cerámicas de baja calidad; San Julián, los Arcos, el Carmen y las Cuevas del Siete, la Carretera de Alcañiz, la Carretera de Zaragoza, el segundo Ensanche, el Casco Histórico, el barrio del Jorgito, el Polígono Industrial y la Ciudad Escolar, con una calidad media-baja; el primer Ensanche de calidad media alta; y las Viñas, La Vega, la Fuenfresca y la Muela con una buena calidad (Figura 1).

Para valorar la calidad del equipamiento urbano hemos utilizado la iluminación, el asfaltado, la limpieza de las calles, y el cuidado de las zonas verdes. La ponderación que se ha dado ha sido de 0,35 a la iluminación; 0,25 al asfaltado, 0,25 a la limpieza de las calles y 0,15 al cuidado de las zonas verdes. Todas ellas son ponderaciones subjetivas que pueden variar según la preferencia de los ciudadanos, como demuestra el sondeo de opinión realizado para establecer la ponderación. Al cuidado de zonas verdes se le ha asignado una menor ponderación porque para poder valorarse primero tiene que haber zona verde, con lo cual este valor distorsionaría mucho la calidad de los equipamientos alli donde no haya zona verde. Los resultados obtenidos se muestran en el cuadro VI.3. 
Cuadro VI.3. Valoración según indicadores de equipamientos

\begin{tabular}{lccccccccc}
\hline $\begin{array}{l}\text { DISTRITOS/ } \\
\text { VARIABLES }\end{array}$ & 1 & 2 & 3 & 4 & & & & & \\
\hline C. Histórico & 3 & 2 & 2 & 2 & 0,75 & 0,70 & 0,50 & 0,30 & 2,25 \\
Arrabal & 3 & 2 & 2 & 0 & 0,75 & 0,70 & 0,50 & 0,00 & 1,95 \\
San Julián & 3 & 2 & 2 & 0 & 0,75 & 0,70 & 0,50 & 0,00 & 1,95 \\
1er Ensanche & 3 & 3 & 2 & 3 & 0,75 & 1,05 & 0,50 & 0,45 & 2,00 \\
$2^{\circ}$ Ensanche & 3 & 2 & 2 & 3 & 0,75 & 0,70 & 0,50 & 0,45 & 2,40 \\
Los Arcos & 3 & 2 & 2 & 0 & 0,75 & 0,70 & 0,50 & 0,00 & 1,95 \\
Ctra. Alcañiz & 3 & 2 & 2 & 1 & 0,75 & 0,70 & 0,50 & 0,15 & 2,10 \\
Carmen/Cueva & 2 & 2 & 2 & 0 & 0,50 & 0,70 & 0,50 & 0,00 & 1,70 \\
Ctr. Zaragoza & 3 & 3 & 2 & 2 & 0,75 & 1,05 & 0,50 & 0,30 & 2,60 \\
C. Escolar & 3 & 2 & 2 & 3 & 0,75 & 0,70 & 0,50 & 0,45 & 2,40 \\
Las Viñas & 2 & 1 & 2 & 1 & 0,50 & 0,35 & 0,50 & 0,15 & 1,50 \\
P. Muela & 2 & 2 & 2 & 1 & 0,50 & 0,70 & 0,50 & 0,15 & 1,85 \\
B Jorgito & 3 & 2 & 2 & 0 & 0,75 & 0,70 & 0,50 & 0,00 & 1,95 \\
La Vega & 3 & 2 & 2 & 1 & 0,75 & 0,70 & 0,50 & 0,15 & 2,10 \\
Fuenfresca & 3 & 3 & 3 & 2 & 0,75 & 1,05 & 0,75 & 0,30 & 2,85 \\
P.Industrial & 2 & 2 & 2 & 1 & 0,50 & 0,70 & 0,50 & 0,15 & 1,85 \\
C San Blás & 1 & 1 & 1 & 1 & 0,25 & 0,35 & 0,25 & 0,15 & 1,00 \\
Cerámicas & 1 & 3 & 0 & 1 & 0,25 & 1,05 & 0,00 & 0,15 & 1,45 \\
\hline
\end{tabular}

Variables: 1) asfaltado; 2) iluminación; 3) limpieza de las calles; 4) cuidado de las zonas verdes.

Los resultados obtenidos se han agrupado en cuatro intervalos:
De 0 a 1,5 baja calidad
De 1,6 a 2 calidad media-baja
De 2,1 a 2,5 calidad media-alta
De 2,6 a 3 alta calidad

Así los resultados obtenidos muestran una baja calidad de equipamientos en la carretera de San Blás y en las Cerámicas; una calidad media-baja en el Arrabal, San Julián, los Arcos, el Carmen y las Cuevas del Siete, las Viñas, la Muela, el barrio del Jorgito, y el Polígono Industrial; una calidad media-alta en el Casco Histórico, el segundo Ensanche, la Carretera de Alcañiz, la Vega y la Ciudad Escolar; y una calidad alta la encontramos en el primer Ensanche, la carretera de Zaragoza y la Fuenfresca (Figura 1).

Como vemos, dentro de un mismo barrio se entremezclan distintas calidades en distintos aspectos, aunque muestran unas claras tendencias en los barrios más desfavorecidos que oscilan entre calidades bajas o medias-bajas en todos los aspectos; mientras que los mayores contrastes 
entre unos y otros aspectos se presentan sobre todo en el Pinar de la Muela y en las Viñas.

Para la valoración global hemos tomado el resultado de las valoraciones parciales anteriores con la denominación de medio natural, edificación y equipamientos; y se han añadido tres nuevas variables, el nivel socioprofesional, el asentamiento de industrias y los servicios. En esta última fase las ponderaciones seleccionadas han sido las siguientes: al medio natural 0,30 , a la edificación 0,25 , a los equipamientos 0,15 , al nivel socioprofesional 0,10 , a las industrias 0,05 y a los servicios 0,15 . Los resultados obtenidos pueden verse en el cuadro VI.4

\section{Cuadro VI.4. Valoración global}

\begin{tabular}{llllllllllllll}
\hline $\begin{array}{l}\text { DISTRITOS/ } \\
\text { VARIABLES }\end{array}$ & 1 & 2 & 3 & 4 & 5 & 6 & & & & & \\
\hline C.Históric & 1 & 2 & 3 & -2 & 3 & 2 & 0,30 & 0,50 & 0,45 & $-0,10$ & 0,30 & 0,30 & 1,75 \\
Arrabal & 1 & 1 & 1,5 & -1 & 1 & 2 & 0,30 & 0,25 & 0,22 & $-0,05$ & 0,10 & 0,30 & 1,12 \\
San Julián & 1 & 2 & 1 & -1 & 1 & 2 & 0,30 & 0,50 & 0,15 & $-0,05$ & 0,10 & 0,30 & 1,30 \\
$1^{\circ}$ Ensanche & 3 & 2 & 2,5 & -1 & 2 & 3 & 0,90 & 0,50 & 0,37 & $-0,05$ & 0,20 & 0,45 & 2,37 \\
$2^{\circ}$ Ensanche & 3 & 2 & 2 & -2 & 2 & 2 & 0,90 & 0,50 & 0,30 & $-0,10$ & 0,20 & 0,30 & 2,10 \\
Los Arcos & 1 & 2 & 1 & -1 & 1 & 2 & 0,30 & 0,50 & 0,15 & $-0,05$ & 0,10 & 0,30 & 1,30 \\
C. Alcañiz & 2 & 2 & 1 & -2 & 1 & 2 & 0,60 & 0,50 & 0,15 & $-0,10$ & 0,10 & 0,30 & 1,55 \\
Carmen/Cue & 1 & 2 & 1 & -1 & 1 & 2 & 0,30 & 0,50 & 0,15 & $-0,05$ & 0,10 & 0,30 & 1,30 \\
C. Zaragoza & 2 & 2 & 2 & -2 & 2,5 & 3 & 0,60 & 0,50 & 0,30 & $-0,10$ & 0,20 & 0,45 & 1,95 \\
C. Escolar & 3 & 2 & 2 & 0 & 2 & 2 & 0,90 & 0,50 & 0,30 & 0,00 & 0,20 & 0,30 & 2,20 \\
Las Viñas & 3 & 3 & 3 & 0 & 0 & 1 & 0,90 & 0,75 & 0,45 & 0,00 & 0,00 & 0,15 & 2,25 \\
P.Muela & 3 & 3 & 3 & 0 & 0 & 2 & 0,90 & 0,75 & 0,45 & 0,00 & 0,00 & 0,30 & 2,40 \\
$B^{0}$ Jorgito & 1 & 2 & 2 & 0 & 0 & 2 & 0,30 & 0,50 & 0,30 & 0,00 & 0,00 & 0,30 & 1,40 \\
La Vega & 2 & 3 & 2 & -3 & 0 & 1 & 0,60 & 0,75 & 0,30 & $-0,15$ & 0,00 & 0,15 & 1,65 \\
Fuenfresca & 3 & 2 & 2,5 & 0 & 1 & 3 & 0,90 & 0,50 & 0,37 & 0,00 & 0,10 & 0,45 & 2,32 \\
P. Industri. & 1 & 3 & 1 & -3 & 0 & 2 & 0,30 & 0,75 & 0,15 & $-0,15$ & 0,00 & 0,30 & 1,35 \\
C.San Blás & 1 & 1 & 2 & -2 & 0 & 1 & 0,30 & 0,25 & 0,30 & $-0,10$ & 0,00 & 0,15 & 0,90 \\
Cerámicas & 1 & 3 & 1 & -3 & 0 & 1 & 0,30 & 0,75 & 0,15 & $-0,15$ & 0,00 & 0,15 & 1,20 \\
\hline
\end{tabular}

Variables: 1) medio natural; 2) edificación ; 3) nivel socioprofesional; 4)industrias; 5) servicios; 6) equipamientos.

La valoración obtenida se ha clasificado en cuatro grupos:

- baja calidad ambiental ............................valores inferiores a 1,30.

- media-baja calidad ambiental..................entre 1,31 y 1,80 .

- media-alta calidad ambiental...................entre 1,81 y 2,30

— alta calidad ambiental ............................valores superiores a 2,31 
Según estos valores, nos encontramos que los barrios con una baja calidad ambiental son el Arrabal, San Julián, Los Arcos, el Carmen/Cuevas del siete, San Blás, y las Cerámicas. Una calidad ambiental media-baja se da en el Casco Histórico, Carretera de Alcañiz, barrio del Jorgito, La Vega, y el Polígono Industrial. Una calidad media-alta hay en el segundo Ensanche, la Carretera de Zaragoza, la Ciudad Escolar y las Viñas. Solamente tres barrios cuentan con una buena calidad ambiental según los indicadores utilizados: el primer Ensanche, el Pinar de la Muela y la Fuenfresca (Figura 2).

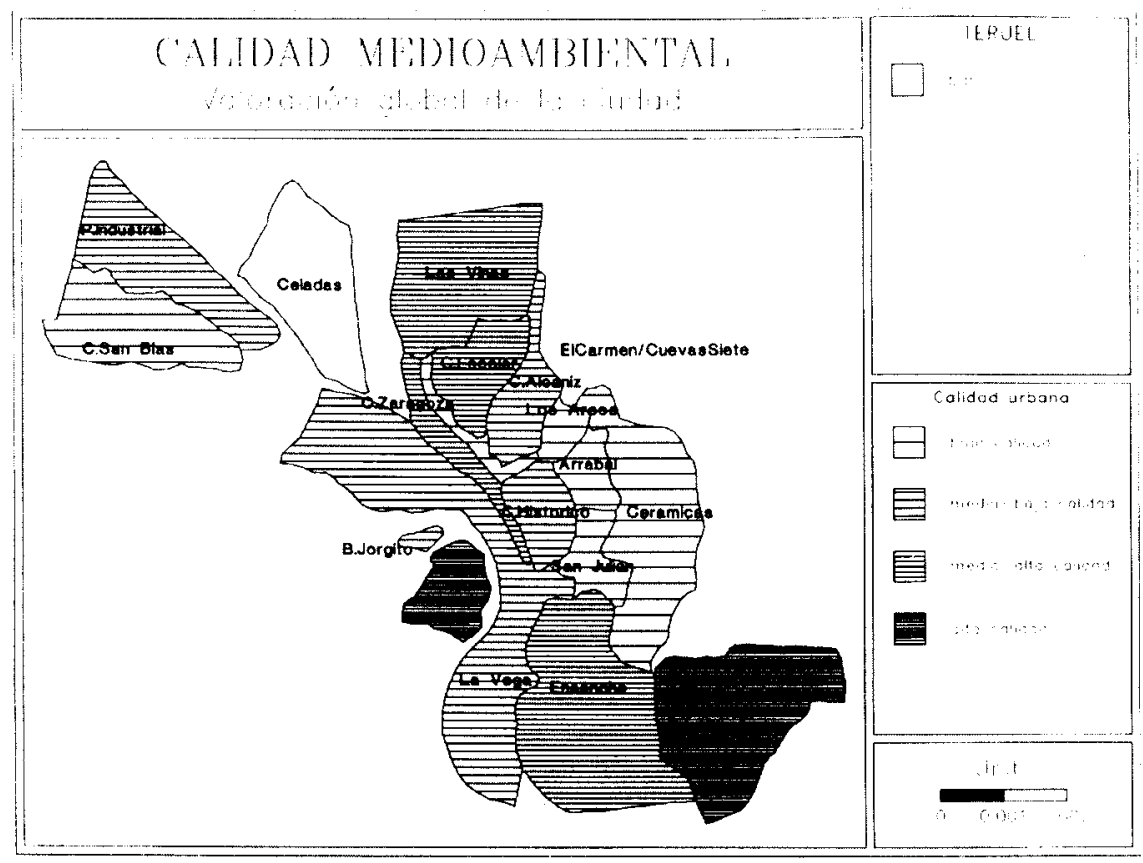

Figura 2. El mapa muestra la valoración relativa entre los distintos barrios de Teruel.

\section{CONCLUSIONES}

Teruel es, como vemos, una ciudad muy heterogénea en cuanto a calidad del medio urbano, con grandes contrastes, que deben reducirse dedicando una especial atención a las áreas degradadas, que repercuten en el ambiente global de la ciudad. 
Ante estos hechos cabe preguntarse ¿para qué ha servido hasta ahora el planeamiento urbano? Su continuada actuación tendente unas veces a transformar la sociedad con un ideal alternativo de ciudad futura que no se correspondía con la realidad; en otras, el planeamiento se ha convertido en un instrumento para legalizar operaciones ya consolidadas o previamente acordadas que eran ilegales a todas luces; $y$ en otras ocasiones ha dejado vía libre al funcionamiento de la ciudad, hecho que favorece a unos y perjudica a otros, siendo los favorecidos los que ya lo están y los perjudicados los que ya lo estaban.

Hoy día, las nuevas líneas de actuación deben asumir una perspectiva global por lo variado de los objetivos que abarcan, desde la regeneración paisajistica hasta la elevación de los servicios, pasando por la conectividad física de los distintos barrios. Como señala el profesor Alonso Vergara Gómez: "Con respecto al marco urbano, es importante asumir una mayor preocupación por la escena urbana, por la imagen de la ciudad, por la morfología, por el espacio urbano y por todo lo que implique una apropiación y disfrute integral de la ciudad por los propios ciudadanos, sin que otro tipo de consideraciones tenga más fuerza que el objetivo de dotar de la máxima calidad a la vida urbana de la ciudad. Paralelamente, es imprescindible una mayor atención a la conservación, rehabilitación y puesta en valor del medio físico".

Pero de hecho, el funcionamiento integrado del sistema ecológico urbano, que nadie discute a nivel teórico, aún dista mucho de hallarse plenamente incorporado a los documentos de planeamiento y a las políticas urbanas. Siguen prevaleciendo actitudes sectoriales, impuestas por la urgencia de los problemas a resolver, o por la rentabilidad económica, o por las políticas que se quiera alcanzar. En este sentido, el programa Hombre y Biosfera de la UNESCO ha intentado aplicar un enfoque ecológico para las ciudades: concibe la ciudad como un ecosistema en cuanto a estructura y funcionamiento, incluyendo componentes tanto bióticos como abióticos. Se trata de abordar la realidad urbana desde presupuestos globales y no sectoriales, como pueden ser los planteamientos para la intervención ambiental en determinados barrios desfavorecidos de las áreas consolidadas.

En aras a la consecución de un término municipal más atractivo y habitable, se debería prestar una especial atención a la adopción de medidas concretas que propicien mejoras en la calidad ambiental. Entre otras cabría destacar las siguientes:

- La disposición de ordenanzas municipales y la vigilancia de su cumplimiento, en lo que se refiere al aspecto exterior de las edificaciones. 
- Una mayor diligencia en el cuidado y limpieza de los espacios públicos.

- En los barrios más degradados de la ciudad, Casco Histórico, Arrabal, Cuevas del Siete, el Carmen y San Julián -además de las recientes y positivas medidas tomadas en pro de su conservación, restauración y rehabilitación, como consecuencia de su declaración, en el año 1997, por la Diputación General de Aragón como Zona de Rehabilitación Preferente- habría que conseguir la ampliación e implantación de espacios públicos destinados a jardines, ocio y juegos, bien como resultado de remodelaciones de las zonas más degradadas o mediante la reconversión de los espacios públicos existentes. En el Casco Histórico, donde la mayor parte de los espacios públicos han pasado a ser dominio del automóvil o han sido transformados en "espacios duros" con predominio de los elementos arquitectónicos y la práctica erradicación de la vegetación, urge la puesta en práctica de zonas peatonales, la replantación de algunos espacios, y la recuperación para espacio público de pequeñas áreas de edificación en ruinas. En los otros barrios, particularmente en el Arrabal, es precisa una remodelación de sus amplias zonas degradadas, que vaya acompañada de la generación de nuevos espacios públicos de esparcimiento y ocio.

- En los barrios de menor crecimiento, Ensanche y Carretera de Alcañiz, se deben evitar las actuaciones en los espacios públicos tendentes a la consecución de un minimalismo funcional en favor del automóvil y la desaparición de la vegetación.

- Se precisa completar la replantación de las diversas laderas de espacio público integradas en el casco urbano, pues de ello se derivan múltiples efectos beneficiosos, tanto ambientales como paisajísticos.

- Se requiere una actuación de restauración de terrenos y mejora paisajística en los límites orientales de los barrios de San Julián, Arrabal y Carrel, que presentan un aspecto ruinoso como consecuencia de la existencia de canteras abandonadas de extracción de arcilla.

- La protección de los ríos, riberas y márgenes de la presa de San Blás, que hagan posible su disfrute como lugares de esparcimiento, ocio y contacto con la naturaleza.

- Urge la preparación de un nuevo vertedero que centralice los residuos urbanos y permita llevar a cabo labores de restauración en el existente, así como en los múltiples vertederos y escombreras que se sitúan en la periferia de la ciudad - cerro Santa Bárbara, Arrabal, San Julián, inmediaciones del hipermercado $e$ instalaciones deportivas y feriales, etc.- , de 
manera que se eviten sus negativos efectos ambientales y paisajísticos. En este mismo sentido habría que solventar los molestos impactos originados por los cementerios de automóviles de la carretera de Zaragoza y de la cuesta del Carrajete.

- Los problemas originados como consecuencia del paso de las carreteras por el casco urbano y sus proximidades, es de esperar que queden resueltos en buena medida cuando se construyan las proyectadas variante norte y autovía Somport-Sagunto.

\section{BIBLIOGRAFIA}

ANALES DE GEOGRAFIA DE LA UNIVERSIDAD COMPLUTENSE, $n ¹ 5$ : Homenaje al profeSOr D. JOAQUín BOSQUE MAUREL. "Ciudad y Medio Ambiente». Madrid 1995. 840 pág.

ChUECA Goltia, F. (1977): "La destrucción del legado urbanístico español». Madrid. Espasa Calpe. 381 págs.

HARVEY, S. Perloff (1973): "La calidad del medio ambiente urbano"Barcelona. Oikos-Tau 342 págs.

LOPEZ GOMEZ, A. et alii (1993): “El clima de las ciudades españolas». Madrid. Cátedra.

MARTINEZ LOPEZ, F y otros (1991): “Macroinvertebrados y calidad de aguas del río Guadalaviar y cuenca del río Mijares (Teruel). Revista del Instituto de Estudios Turolenses». Teruel, págs. 115-160.

Puebla Remacha, M.P. y otros (1990): “Estudios de los vertidos industrial y urbano de Teruel. Propuesta de mejoramiento del rio Turia". Revista del Instituto de Estudios Turolenses. Teruel, págs. 345-369.

VERGARA GOMEZ, A. (1993): "Cultura urbana y cultura del territorio. Los retos del urbanismo del siglo XX|». Ciudad y Territorio, nº 95-96, pág. 248. 